\title{
Comparing high-resolution satellite and GatorEye UAV lidar data for trail mapping in mixed pine and oak forests in central Florida using a participatory approach
}

\author{
Mabel Cesarina Báez ${ }^{1}$, Angélica María Almeyda Zambrano ${ }^{2}$, Beatriz Lopez Gutierrez ${ }^{3}$, Gretchen Stokes ${ }^{4}$, \\ Jaime Chavez ${ }^{5}$, Pamela Montero-Alvarez ${ }^{4}$, Ana Oliveira Fiorini ${ }^{4}$, Diego Garcia Olaechea ${ }^{6}$, Ben Wilkinson ${ }^{1}$, \\ Eben North Broadbent ${ }^{3 *}$
}

1 School of Forest Resources and Conservation, University of Florida, Gainesville, Florida 32611; mabelbaez@ufl.edu (M.C.B.); benew@ufl.edu (B.W.)

2 Center for Latin America Studies, University of Florida, Gainesville, Florida 32611; aalmeyda@ufl.edu (A.M.A.Z.)

3 Spatial Ecology and Conservation Lab, School of Forest Resources and Conservation, University of Florida, Gainesville, Florida 32611; eben@ufl.edu (E.N.B.); blopezgutierrez@ufl.edu (B.L.G.)

4 School of Natural Resources and Environment, University of Florida, Gainesville, Florida 32611; anacarolinfiorini@gmail.com (A.O.F.); gstokes@ufl.edu (G.S.); pamela.monteroa@ufl.edu (P.M.A.)

5 Department of Agricultural and Biological Engineering, University of Florida, Gainesville, Florida 32611; jamesvic@ufl.edu (J.C.)

6 Department of Wildlife Ecology and Conservation, University of Florida, Gainesville, Florida 32611; diego.garcia@ufl.edu (D.G.O.)

* Correspondence: eben@ufl.edu (E.N.B.)

\begin{abstract}
Trail detection in mixed canopy ecosystems has important implications for forest management, monitoring, and conservation, although active sensor technology for sub-canopy trail detection is still developing. In order to assess the effectiveness of UAV(Unmanned Aerial Vehicle)-borne lidar (light detection and ranging) data for small trails $(<2.5 \mathrm{~m}$ width) in mixed forest canopy cover, we collected lidar data and trail characteristics (canopy cover and trail width) and created a high definition surface model map from the resulting lidar data, and also a high-resolution satellite imagery map using Google Earth. Through participatory mapping methods, seven respondents with limited prior geospatial experience completed a rapid identification of trails on both maps. Respondents' trails were georeferenced in order to compare the rate of detectability between maps. We found greater detection on the lidar-derived map compared to the Google Earth map. Detectability in Google Earth maps was positively correlated with wider trails and trials with lower canopy. In lidar maps, trail detectability increased with wider trails, but canopy cover had no effect on detection rates. Our data indicate that a mixed-method approach that combines UAV-mounted lidar with high-resolution satellite imagery and participatory mapping increases rapid detection rates of small trails under varying canopy cover and trail widths.
\end{abstract}

Keywords: Unmanned Aerial Vehicles; trail mapping; conservation; forestry; canopy cover

\section{Introduction}

Remote sensing techniques have many applications for natural resource management and conservation, such as habitat analysis, measuring forest carbon stocks, protected area delineation, landscape modeling, and human impact surveys (e.g., logging, mining) [1-4]. Currently, among the remote sensing methodologies, light detection, and ranging (lidar) is the most accurate tool for quantifying forest structure characteristics [5]. These measures are accurate in a wide range of forested ecosystems and even have applications in urban settings (e.g. [6]). Unlike photogrammetric systems which use passive sensors requiring sunlight to illuminate the area of interest, lidar sensors actively emit 
laser pulses to create three-dimensional point clouds. When point cloud density is high, lidar can be used for measuring tree height, identifying tree species, estimating crown canopy profile, estimating biomass, and generating carbon projections at various spatial scales [5,7-12]. Lidar sensors can be satellite or aircraft-borne, deployed above and below the canopy with unmanned aerial vehicles (UAVs), or ground-based [8,12-13].

Recently, airborne lidar has been used to identify roads in urban and suburban systems $[6,14]$. The identification of roads and trails in forested landscapes, which is essential for natural resource management, is far more difficult due to variable topography and complex forest structure, even for airborne lidar-derived maps [14]. White et al. [14], Lee et al. [15], and Kim and Olsen [16] developed methods to identify roads in forested, topographically complex terrains using airborne lidar; however, these studies only looked at roads more than $2 \mathrm{~m}$ in width. Meanwhile, Espinoza and Owens [17] were able to identify trails less than $2.5 \mathrm{~m}$ with an $85 \%$ accuracy using airborne lidar data. Their analysis, however, excluded sections of old-growth forest with thick canopies [17].

We build upon these research works for the first study that maps trails using UAV lidar-derived maps. UAVs, also referred to as drones, are revolutionizing the field of remote sensing, particularly for applications in wildlife monitoring, conservation, and forestry [18-19]. UAVs require low starting and operational costs, provide a platform on which high-resolution sensors can be mounted, and allow for the rapid collection of data across spatial and temporal scales even in challenging landscapes [18-20]. UAVs are also making data more readily available to primary users, with the rapid development of user-friendly interfaces that allow for easy processing and interpretation of data [19]. While some UAV-mounted sensors can provide instantaneous data (e.g., thermal sensors), others require post-processing (e.g., lidar). Currently, lidar data often relies on a deep knowledge of data processing.

Our comparison of UAV lidar data and high-resolution satellite images involves the manual identification of small $(<2.5 \mathrm{~m})$ trails under various canopy densities in mixed pine and oak forest in central Florida. Local students identified trails through a participatory mapping exercise. Participatory mapping facilitates trail identification because automation of this process requires specialized software and extensive parametrization for each new location [14,17]. It takes advantage of the brain's ability to identify patterns and allows for the input of contextual knowledge. This brings local context and knowledge for simpler and more effective trail identification and helps close the gap between primary field users and experts.

We hypothesize that students in our participatory mapping exercise will identify more trails using our lidar-derived map compared to our high-resolution satellite map as the forest canopy gets more complex. This is because we will be able to "eliminate" canopy points from our point clouds in this case. We expected that satellite imagery would be more effective in low canopy areas where trails can be easily identified due to soil wear.

\section{Materials and Methods}

\subsection{Study Area}

The University of Florida's Natural Area and Outdoor Teaching Laboratory (NATL) is a 60 -acre experimental forest located in Southwest Gainesville, FL $\left(29.6354^{\circ} \mathrm{N}, 82.3677^{\circ}\right.$ W). NATL is comprised of two contiguous tracts (NATL-East, NATL-West) of upland forest ecosystems characteristic of North Florida: hammock, upland pine, and old-field succession. Mean tree height across forested areas is $23 \mathrm{~m}$, with a dense understory often comprised of saw palmetto (Serenoa repens) or woody shrubs (e.g., Crataegus uniflora). NATL also has a diverse wetland habitat, including a 9-acre marsh that drains into a pond and a 3-acre ecologically engineered retention basin (Figure 1). The diversity of forest ecosystems within NATL makes it an ideal test site for a trail detection study. There are $6,665 \mathrm{~m}$ of marked trails distributed across NATL. These trails are primarily 
used by pedestrians for research (e.g., wildlife tracking, forestry measurements), recreation (e.g., walking, bird watching), and maintenance, which sometimes requires the use of small vehicles (e.g., equipment repairs).
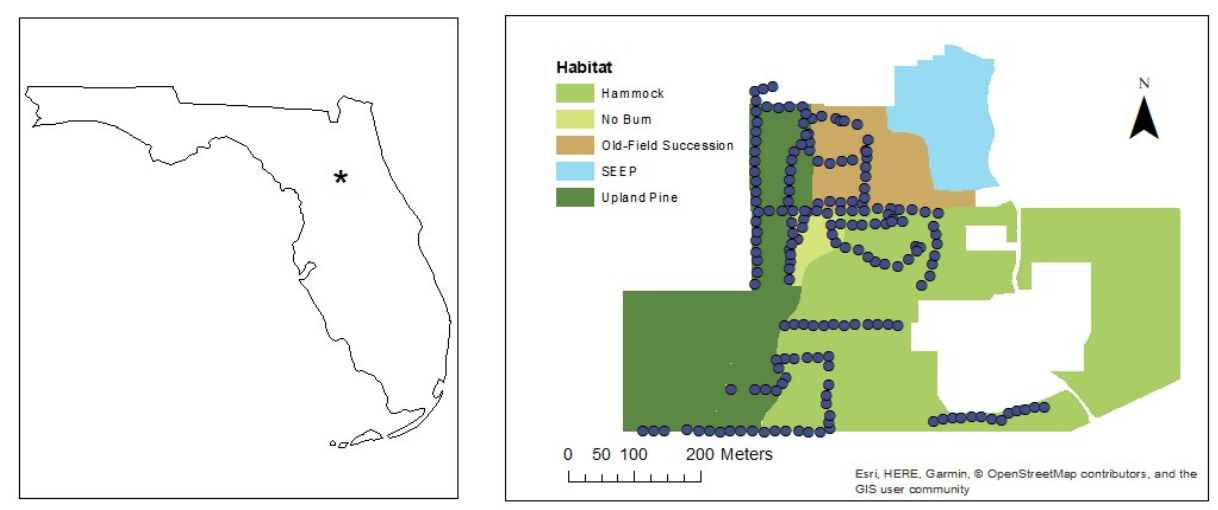

Figure 1. Map and land use of the Natural Area Teaching Laboratory in the University of Florida, Gainesville, Florida. Blue dots represent trail area where width and canopy cover were collected.

\subsection{Methods}

We integrated UAV lidar cloud points, and trail characteristics field data in order to assess the effectiveness of UAV lidar data in identifying small $(<2.5 \mathrm{~m})$ trails under various canopy densities using participatory mapping of trails.

\subsubsection{UAV lidar}

All lidar data in this study were collected in NATL on 27 February 2018 using the GatorEye Unmanned Flying Laboratory (GE-UFL). The GE-UFL is a sensor suite that includes a Headwall photonics VNIR 270 spectral band hyperspectral sensor, and high-resolution RBG camera, and a Phoenix Aerial VLP-16 dual-return lidar sensor (600,000 returns/ second). It is geolocated using dual-frequency point positioning and post-processed kinematic algorithms relative to a base station to produce point clouds with approximately $10 \mathrm{~cm}$ accuracy (for detailed information, refer to www.gatoreye.org). We conducted five UAV missions (flight time $=90$ minutes) in optimal weather conditions (i.e., low wind, sunny) between 11 am and 3 pm (Table 1). Using the resulting LiDAR data (Figure 2), we classified ground points using LASTools software [21]. Based on the ground-classified points, we extracted all lidar points using a three-meter threshold, above or below ground level. Subsequently, we rasterized the point cloud at a resolution of $10 \mathrm{~cm}$ using the highest point in each raster cell. Finally, a sun inclination and azimuth of 40 and 40 degrees illuminated the corresponding surface model map (Figure 3). We found this processing workflow produced the most surface contrast, which is favorable for the manual identification of trails.

Table 1. Flight and Sensor Parameters

\begin{tabular}{lr}
\hline LiDAR Survey Parameters & \\
\hline Altitude (m AGL) & 60 \\
Flight Speed (m/s) & 6 \\
Transect separation (m) & 20 \\
Transect azimuth Angle & 90 \\
Sampling Density (ppm2) & 520 \\
\hline
\end{tabular}




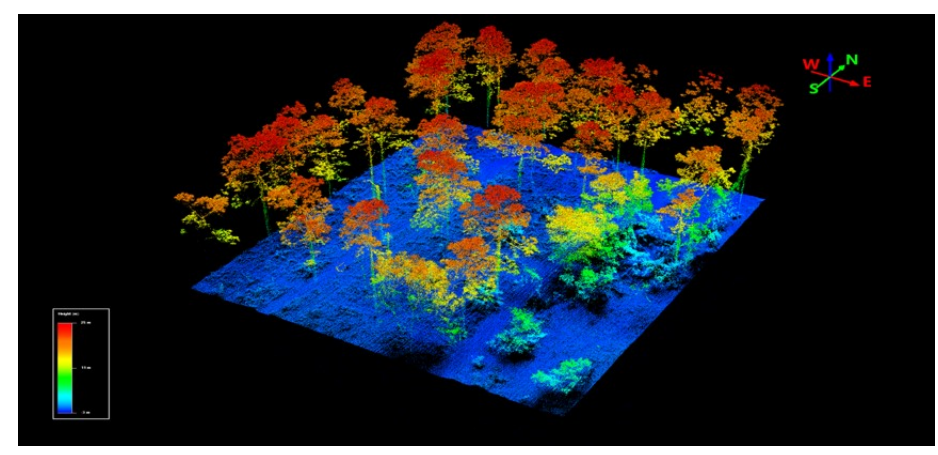

Figure 2. An example portion of the LiDAR point cloud used for this project which was acquired at the University of Florida Natural Area and Outdoor Teaching Laboratory (NATL), in Gainesville, FL.

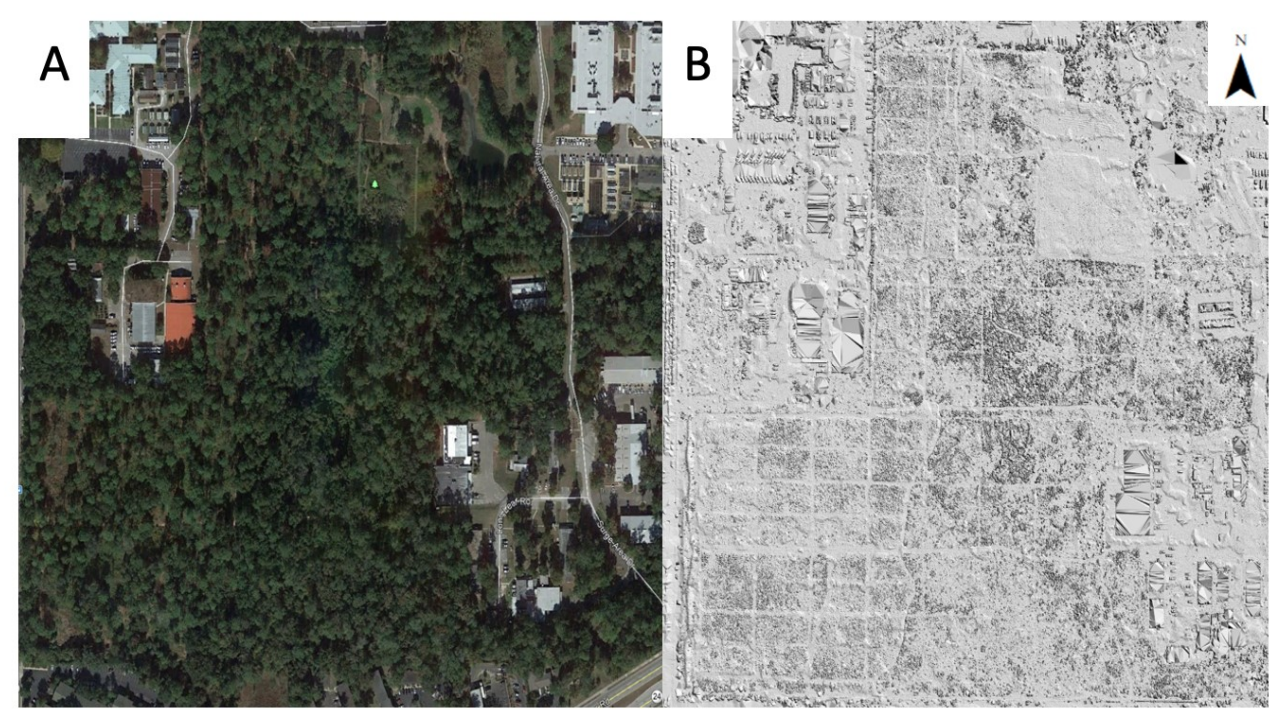

Figure 3. Google Earth (A) and Lidar-derived (B) maps of the University of Florida's Natural Area Teaching Laboratory in Gainesville, Florida, presented to each respondent for rapid (2 minutes) trail identification.

\subsubsection{Trail Surveys}

We measured a total of 179 field points across all ecosystems in NATL except SEEP. For every $15 \mathrm{~m}$ along these trails, we recorded percent canopy cover and trail width. Mean canopy cover on the trails was $74 \%$ (range $=2$ to $100 \%$ ). The average trail width was $1.5 \mathrm{~m}(0.5$ to $3 \mathrm{~m})$. Geographic locations were marked using a Trimble Geo7x, GeoXH, and Garmin GPS Map 64. GPS coordinates were differentially corrected using Trimble Pathfinder Software. The width was measured as the perpendicular distance across the visible trail surface. Canopy cover was measured from the recorded midpoint of the trail using a Forestry Suppliers Spherical Crown Densiometer (Concave Model C) [22].

\subsubsection{Participatory Mapping of Trails}

Graduate students at the University of Florida $(n=7)$, with no prior knowledge of the study area, had two minutes to manually delineate the trail system in each of the two maps. The first map was a 50- $\mathrm{cm}$ resolution Google Earth satellite image of the study area (image date 11/19/2016) (Figure 3). The second was a post-processed image of collected UAV lidar cloud points rasterized using the methods in 2.2.1. We imported, digitized, and combined participant's maps into ESRI ArcMap [23] to create two composite maps of trails identified using our GE and lidar maps. To account for tracing error, we created a 2 
$\mathrm{m}$ buffer around each line. To create a measure of trail detectability, we isolated and identified which field points fell within the participant's trail buffers. From this, we created a percent detectability based on how many individuals identified each point.

\subsection{Analyses}

In order to examine which map was more efficient for trail identification, we conducted a matched-pairs t-test of points detected using each method. To test the relationship between trail canopy and width with detectability between each mapping method, we used regression analysis. All analyses were conducted in JMP Pro software [24].

\section{Results}

Overall, we found more trails points were identified using the lidar map $(68 \%)$ than the Google Earth map (36\%) $(t(178)=-5.00, p=0.0001)$ (Figure 4). We found a significant relationship between canopy cover and trail width, with lower canopy cover with increasing trail width $(\mathrm{p}=0.01)$.
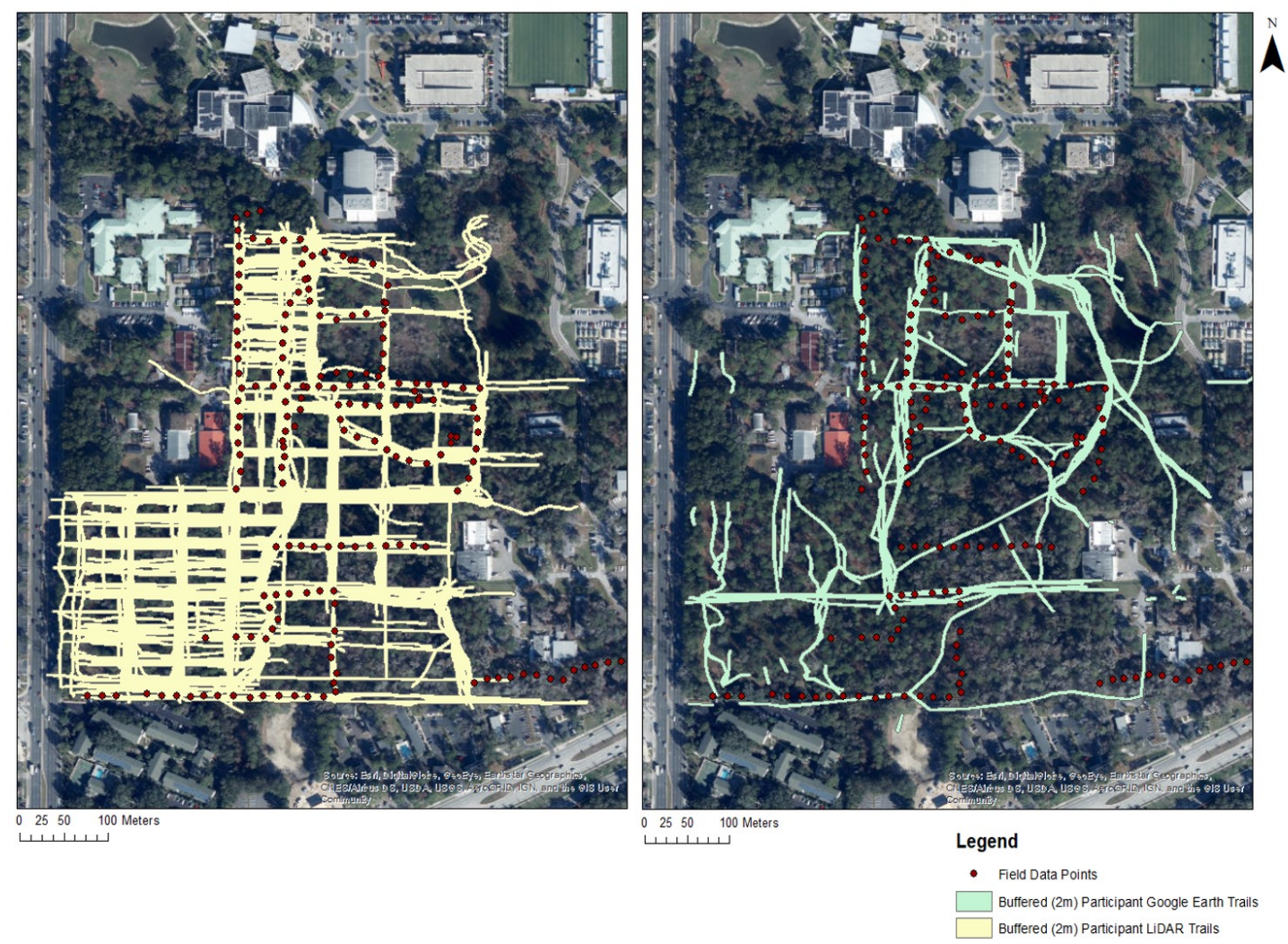

Figure 4. Georeferenced trail lines from participants ( $n=7)$ using UAV lidar derived map (A) and Google Earth map (B) of the University of Florida's Natural Area Teaching Laboratory in Gainesville, Florida. Lines denote trails (buffered 2 m) participants identified.

Most notably, using the lidar maps, we found a negative relationship between detectability and trail width, with less detectability with bigger trails $(\mathrm{p}<0.0001)$. However, we found that canopy density had no significant effect on detectability $(p=0.07)$. There was also no significant mixed effect of canopy density and trail width $(p=0.10)$. Using the Google Earth maps, as expected, detectability was positively associated with trail width, with increased detections of bigger trails $(p=0.008)$. As expected, there was a negative relationship between detectability and canopy density, with lower detectability 
with increasing canopy density $(p=0.01)$. There were no significant mixed effects between detectability of trails in Google Earth and width and canopy density $(p=0.34)$.

\section{Discussion}

Lidar allows scientists to understand physical variation in forested landscapes at a large scale across space and time. However, studies evaluating changes in the understory, such as trails, with airborne sensors or satellite images, remain scarce [16,25]. Of the airborne lidar studies identifying trails, many struggle to identify narrow $(<2.5 \mathrm{~m})$ trails in forested landscapes with thick canopies. In this study, we assessed the effectiveness of UAV lidar data, compared to high-resolution satellite images, in the manual identification of narrow trails under various canopy densities in a mixed pine and oak forest in Natural Area Teaching Laboratory (NATL) at the University of Florida in Gainesville, Florida. Finally, we analyzed which parameters (canopy density and trail width) were associated with trail detectability and how these parameters affected detection in each map.

As expected, we found that participants are able to identify more trails more effectively with lidar maps than Google Earth imagery. Overall, participants correctly identified almost twice as many field points with lidar maps $(68 \%)$ than with Google Earth maps (36\%). This rate of detectability is impressive, given that it was not automated and done within a short time frame. On the other hand, a previous automated study done by Espinoza and Owen's [17] had a greater accuracy rate (85\%), although they excluded a section of old-growth forest with a thick canopy.

Surprisingly, in lidar maps, we found that detectability decreased in bigger trails. We attribute this to the fact that within NATL, the edges in bigger trails were more diffuse. For smaller trails, we found that large trees or shrubs demarcated the edges. Because of the high point density in the lidar data (Figure 2), ground relief remained detailed after parceling the point cloud using a three-meter threshold; thus, canopy density did not affect detectability. The opposite was true for detectability on Google Earth maps, where bigger trails and trails with less canopy density were more easily detected. In open areas, participants were able to detect trails using color cues in the Google Earth maps where even if the grass was short, the green grass was different from the darker, mostly soil, trails. These trails are less distinct in lidar maps as ground points are only slightly lower than the grass-return points.

This pilot study demonstrates that UAV mounted lidar can aid in the quick, manual identification of forest trails - particularly smaller ones-compared to satellite imagery. This has important implications for the evaluation, management, and conservation of natural areas, as the development of illegal trails or changes in established trails can be readily identified using this method. We recommend further studies that include more representative example trails in heterogeneous complex geography (e.g., include creeks and mountains) and other forest ecosystems beyond hammock and pines (e.g., broadleaf). These parameters can affect lidar ground point saturation, which can affect trail detectability. This work also indicates that lidar data can also be used in programs that automatically detect trails, as this may be more effective and precise than our method of rapid human identification. If refined, these methods will be useful for forest applications, military and defense training, agriculture, marking poaching routes, and park trail maintenance, among others.

\section{Conclusion}

UAVs provide a novel and accessible platform for cutting-edge sensors used in remote sensing, such as lidar sensors. Compared to airborne sensors, UAVs allow individuals to study systems at a smaller scale with higher resolution. Our pilot study supplements previous research done on the identification of small trails under various cano- 
pies using airborne lidar and add more insights by integrating UAVs and lidar sensors with participatory mapping. This combination increases the accessibility of these tools for rapid applications across a variety of fields, and we expect to pave the way for more engagement in the near future.

\section{Supplementary Materials: NA.}

Author Contributions: All authors conceived, designed, and performed fieldwork for this study. E.N.B. developed and flew the drone mission, and E.N.B. and A.M.A.Z. conducted LiDAR pre- and post-processing. A.C.F. and B.W assisted E.N.B. with drone preparations and missions. D.G.O., J.V.L, P.M.A., B.L.G., M.B., and G.S. conducted trail measurements. All authors contributed to the analysis, writing of the paper, and manuscript revisions.

\section{Funding: NA.}

Data Availability Statement: Raw lidar data used in this study is available for download from www.gatoreye.org.

Acknowledgments: We thank the staff at NATL, including the 2017 graduate coordinator, Jesh Hong. Funding and support for this study were provided by the Spatial Ecology and Conservation Laboratory and the School of Forest Resources and Conservation and through the McIntire-Stennis program of the USDA.

Conflicts of Interest: The authors declare no conflict of interest.

\section{References}

1. J. Campbell and R. Wynne, Introduction to remote sensing. Guilford Press, 2011.

2. G. P. Asner and J. Mascaro, "Mapping tropical forest carbon: Calibrating plot estimates to a simple LiDAR metric," Remote Sens. Environ., vol. 140, pp. 614-624, 2014.

3. M. Borengasser, W. Hungate, and W. Russell, Hyperspectral Remote Sensing. 2007.

4. D. Pflugmacher, W. B. Cohen, R. E. Kennedy, and Z. Yang, “Using Landsat-derived disturbance and recovery history and lidar to map forest biomass dynamics," Remote Sens. Environ., vol. 151, pp. 124-137, 2014. [

5. D. Pflugmacher, W. B. Cohen, and R. E. Kennedy, “Using Landsat-derived disturbance history (1972-2010) to predict current forest structure," Remote Sens. Environ., vol. 122, pp. 146-165, 2012.

6. J. Han, D. Kim, M. Lee, and M. Sunwoo, “Enhanced road boundary and obstacle detection using a downward-looking LIDAR sensor," IEEE Trans. Veh. Technol., vol. 61, no. 3, pp. 971-985, 2012.

7. T. T. Caughlin, S. W. Rifai, S. J. Graves, G. P. Asner, and S. A. Bohlman, "Integrating LiDAR-derived tree height and Landsat satellite reflectance to estimate forest regrowth in a tropical agricultural landscape," Remote Sens. Ecol. Conserv., vol. 2, no. 4, pp. 190-203, 2016.

8. R. A. Chisholm, J. Cui, S. K. Y. Lum, and B. M. Chen, “UAV LiDAR for below-canopy forest surveys,” J. Unmanned Veh. Syst., vol. 01, no. 01, pp. 61-68, 2013.

9. G. P. Asner, D. E. Knapp, R. E. Martin, R. Tupayachi, C. B. Anderson, J. Mascaro, F. Sinca, K. D. Chadwick, M. Higgins, W. Farfan, W. Llactayo, and M. R. Silman, “Targeted carbon conservation at national scales with high-resolution monitoring," Proc. Natl. Acad. Sci., vol. 111, no. 47, pp. E5016-E5022, 2014.

10. R. Dinuls, G. Erins, A. Lorencs, I. Mednieks, and J. Sinica-Sinavskis, "Tree species identification in mixed baltic forest using LiDAR and multispectral data," IEEE J. Sel. Top. Appl. Earth Obs. Remote Sens., vol. 5, no. 2, pp. 594-603, 2012.

11. L. Wallace, A. Lucieer, C. Watson, and D. Turner, "Development of a UAV-LiDAR system with application to forest inventory," Remote Sens., vol. 4, no. 6, pp. 1519-1543, 2012.

12. D. Roberti, A. De Almeida, S. C. Stark, G. Shao, J. Schietti, W. Nelson, C. A. Silva, E. B. Gorgens, R. Valbuena, D. De, A. Papa, P. Henrique, and S. Brancalion, "Optimizing the Remote Detection of Tropical Rainforest Structure with Airborne Lidar: Leaf Area Profile Sensitivity to Pulse Density and Spatial Sampling."

13. R. A. Rose, D. Byler, J. R. Eastman, E. Fleishman, G. Geller, S. Goetz, L. Guild, H. Hamilton, M. Hansen, R. Headley, J. Hewson, N. Horning, B. A. Kaplin, N. Laporte, A. Leidner, P. Leimgruber, J. Morisette, J. Musinsky, L. Pintea, A. Prados, V. C. Radeloff, M. Rowen, S. Saatchi, S. Schill, K. Tabor, W. Turner, A. Vodacek, J. Vogelmann, M. Wegmann, D. Wilkie, and C. Wilson, "Ten ways remote sensing can contribute to conservation," Conserv. Biol., vol. 29, no. 2, pp. 350-359, 2015.

14. R. A. White, B. C. Dietterick, T. Mastin, and R. Strohman, "forest roads mapped using LiDAR in steep forested terrain," Remote Sens., vol. 2, no. 4, pp. 1120-1141, 2010.

15. H. Lee, K. C. Slatton, and H. Jhee, "Detecting forest trails occluded by dense canopies using ALSM data," Int. Geosci. Remote Sens. Symp., vol. 5, pp. 3587-3590, 2005.

16. A. M. Kim and R. C. Olsen, "Detecting trails in lidar point cloud data," vol. 8379, p. 837906, 2012.

17. F. Espinoza and R. Owens, "Identidying roads and trails hidden under canopy using LiDAR," 2007. 
18. R. Schiffman, “Wildlife conservation: Drones flying high as new tool for field biologists," Science (80-. )., vol. 344, no. 6183, p. 459, 2014.

19. L. Pádua, J. Vanko, J. Hruška, T. Adão, J. J. Sousa, E. Peres, and R. Morais, “UAS, sensors, and data processing in agroforestry : a review towards practical applications," Int. J. Remote Sens., vol. 38, no. 8-10, pp. 2349-2391, 2017.

20. L. Tang and G. Shao, “Drone remote sensing for forestry research and practices,” J. For. Res., vol. 26, no. 4, pp. 791-797, 2015.

21. M. Isenburg, “LAStools- efficient LiDAR processing software.” .

22. G. Strickler, "Use of the densiometer to estimate density of forest canopy on permanent sample plots," PNW Old Ser. Res. Notes, vol. 180, pp. 1-5, 180, 1959.

23. ESRI, "ArcGIS: Version 10.5," 2017.

24. "JMP Pro." SAS Institute Inc., Carry, NC.

25. A. Karatolios and K. Prokopios, "Extracting hidden trails and roads under canopy using LIDAR," Naval Postgraduate School, Monterey CA, 2008.

26. R. S. DeFries, M. Hansen, J. R. G. Townshend, and R. Sohlberg, “Global land cover classifications at 8 km spatial resolution: The use of training data derived from Landsat imagery in decision tree classifiers," Int. J. Remote Sens., vol. 19, no. 16, pp. 3141-3168, 1998. 